\title{
Análise da microdureza Vickers de zircônia Y-TZP pré-sinterizada para a usinagem e posterior aplicação como copings
}

\author{
Vickers microhardness analysis of pre-sintered \\ Y-TZP zirconia for machining and subsequent \\ application as copings
}

Lieca Hassegawa Kavashima ${ }^{1}$, Matheus Gallo Sanches ${ }^{1}$, Edson Antonio Capello Sousa ${ }^{1}$, Carla Müller Ramos ${ }^{2}$, Ana Flávia Sanches Borges ${ }^{2}$, Carlos Alberto Fortulan ${ }^{3}$, Cesar Renato Foschini ${ }^{1}$

\footnotetext{
${ }^{1}$ Departamento de Engenharia Mecânica - FEB - UNESP, Bauru, SP, Brasil e-mail: lie-ca@hotmail.com

${ }^{2}$ Departamento de Dentística, Endodontia e Materiais Odontológicos - FOB - USP, Bauru-SP, Brasil

${ }^{3}$ Laboratório de Tribologia e Compósitos - EESC - USP, São Carlos, SP, Brasil
}

\begin{abstract}
RESUMO
A zircônia Y-TZP é muito utilizada na confecção de coping como material estrutural para próteses odontológicas devido à sua cor branca/perolada opaca e resistência mecânica. Estas próteses são confeccionadas por usinagem com apoio de um software de CAM (Computer Aided Manufacturing), a partir de blocos prensados e pré-sinterizados. A pré-sinterização consiste em aquecer os blocos compactados a altas temperaturas, com uma taxa lenta de aquecimento até o início da formação dos pescoços entre as partículas, quando proporciona dureza/resistência e usinabilidade. Uma contradição, pois a dureza/resistência mecânica é necessária para proporcionar estabilidade as forças de corte, mas se muito duro, pode dificultar a usinagem então se deve encontrar uma faixa de compromisso de temperatura entre estabilidade e usinabilidade. Dessa forma, o objetivo do presente trabalho foi investigar a relação entre a microdureza e temperatura de pré-sinterização da zircônia Y-TZP e seu comportamento ao ser submetido à usinagem. $\mathrm{O}$ ensaio de microdureza foi escolhido como representante de resistência mecânica por ser não destrutivo e de resultado imediato. O pó de zircônia experimental foi submetido à prensagem uniaxial seguido de prensagem isostática a frio à $200 \mathrm{MPa}$ e présinterização entre $900^{\circ} \mathrm{C}$ e $950^{\circ} \mathrm{C}$. Os blocos foram cortados e submetidos ao teste de estabilidade na usinagem e ensaio de microdureza Vickers sendo os dados submetidos ao teste de "normalidade" de AndersonDarlin com $\mathrm{p}>0,05$. Foi utilizada ANOVA de um fator com o teste de Tukey-Kramer para verificar as diferenças entre as médias dos grupos. A investigação demonstrou que a diferença de $50^{\circ} \mathrm{C}$ na temperatura de pré-sinterização, acima de $900^{\circ} \mathrm{C}$ afetou significativamente a microdureza, contudo os tempos de patamar $2 \mathrm{e}$ 4 horas não a influenciou significativamente. A maior temperatura de pré-sinterização dos grupos experimentais testados possibilitou um melhor comportamento na usinagem com o apoio de um software CAM, sendo a microdureza similar ao material comercial ZL.
\end{abstract}

Palavras-chave: Y-TZP, copings, pré-sinterização, microdureza, usinagem, CAD/CAM

\section{ABSTRACT}

Y-TZP zirconia is widely used in the manufacture of coping as a structural material for dental prosthesis due to its opaque white/pearl color and mechanical strength. Dentures are made by machining with cam (computer aided manufacturing) software support, from pressed and pre-sintered blocks. Pre-sintering consists of heating the compressed blocks to high temperatures, with a slow heating rate until the neck formation among particles, when this provides hardness/strength and machinability. There is a contradiction, because the mechanical hardness/strength is necessary to provide stability to the cutting forces, but if it is very hard, machining can be difficult, therefore must be found a compromise zone of temperature between stability and machinability. In this way, the purpose of this study was to investigate the relationship between microhardness and the pre-sintering temperature of experimental Y-TZP zirconia and its behavior to be subjected to machin- 
ing. The microharness testing was chosen as representative of mechanical strength once it is nondestructive and with immediate result. The experimental zirconia powder was subjected to uniaxial pressing, subsequently cold isostatic pressing of $200 \mathrm{MPa}$ and pre-sintering between $900^{\circ} \mathrm{C}$ and $950^{\circ} \mathrm{C}$. Blocks were cut and subjected to machining stability testing and vickers microhardnes testing which the data were subjected to the anderson-darlin ( $p>0.05)$ test for "normality". Anova's "one way" with tukey-kramer test was used to verify the differences among the means of groups. The investigation showed that the difference of $50^{\circ} \mathrm{C}$ in presintering temperature above $900^{\circ} \mathrm{C}$ significantly affect the hardness, whereas 2 to 4 hour time did not significantly affect material microhardness. The highest pre-sintering temperature of the tested experimental groups enabled better performance in machining with cam software support, which provides similar microhardness to commercial ZL material.

Keywords: Y-TZP, copings, pre-sintering, microhardness, machining, CAD/CAM.

\section{INTRODUÇÃO}

A confecção de implantes odontológicos de materiais cerâmicos, como a zircônia Y-TZP está em crescimento devido à hipersensibilidade de alguns pacientes a materiais metálicos, biossegurança e o desejo por melhoria na estética. Dessa forma busca-se a substituição das próteses metálicas por cerâmicos de igual ou superior resistência mecânica. Com a introdução da tecnologia CAD/CAM (Computer Aided Design/Computer Aided Manufacturing), foi possível melhorar a produtividade, uma vez que a tecnologia odontológica convencional não oferece a mesma facilidade em produzir o implante na forma desejada. Assim blocos de Y-TZP compactados ou pré-sinterizados (blanks) são usinados para proporcionar a forma do implante e posteriormente são sinterizados para promover a resistência mecânica solicitada em um ambiente bucal. Os implantes odontológicos de zircônia mais produzidos são coroas, pontes e pilares[1]. A coroa normalmente é composta por uma estrutura suporte opaca com o formato ou morfologia do dente, denominada coping, que pode ser constituída por zircônia e então recoberta por camadas de porcelana ou vitrocerâmica (a exemplo da vitrocerâmica à base de dissilicato de lítio) para mimetizar a cor e brilho do dente [2]. O pilar (zircônia) também conhecido como abutment tem a função de fixar a coroa no parafuso que será introduzido no osso para a fixação da prótese [3].

A zircônia é muito utilizada na confecção de coping devido à sua cor branco/perolado opaca e alta resistência mecânica. Um bloco de zircônia pré-sinterizado é inserido em um centro de usinagem, o qual segue um programa CNC (Comando Numérico Computadorizado) elaborado com o apoio de um programa de computador do tipo CAM. O desenho do dente é obtido por digitalização do dente a ser reparado ou de seus vizinhos, um programa CAD especializado converte, repara as faltas e/ou propõe um sólido 3D. Desta forma, o coping é modelado à partir do sólido 3D sendo usinado de um bloco e posteriormente sinterizado a fim de proporcionar resistência mecânica estrutural [4,5].

A usinagem de produtos próxima à forma final, também conhecida como near-net-shape, é um conceito de manufatura industrial que se preocupa com a redução de etapas de produção, redução de custos e garantia da qualidade do produto para se adquirir a forma final de componentes. Compósitos e cerâmicos sinterizados são caracterizados pela baixa usinabilidade e requer alto custo. Dessa forma o princípio do near-netshape atendido pela usinagem no estado "verde", ou seja, somente compactado, tem alta taxa de remoção, baixo desgaste de ferramenta, consumo energético e elimina passos desnecessários [6].

O coping pode ser considerado de geometria complexa e usinado na forma pré-sinterizada favorece a redução de custo de processo e ainda pode reduzir as distorções na forma final pós sinterização, uma vez que a usinagem ajuda a minimizar os gradientes de densidade oriundos da prensagem [7]. Embora esse tipo de usinagem enfrente algumas dificuldades como fragilidade e dimensão inadequada da prótese devido à retração após sinterização, estas podem ser superadas melhorando-se a qualidade da compactação e dureza, utilização de ferramentas adequadas, composição do agente aglutinante e parâmetros de usinagem [8].

As próteses odontológicas de zircônia Y-TZP normalmente são confeccionadas por usinagem com apoio de um programa de computador do tipo CAM, a partir de blocos prensados pré-sinterizados com dureza o suficiente para o manuseio, mas não prejudiciais à usinagem [9]. A pré-sinterização consiste em aquecer os blocos compactados, à temperaturas cerca de $70 \%$ da temperatura de sinterização, com uma taxa lenta de aquecimento até o início da formação dos pescoços entre as partículas quando proporciona dureza/resistência e usinabilidade. Essas são duas características opostas, pois a dureza é necessária para proporcionar estabilidade no manuseio, mas se muito duro, pode prejudicar a usinagem, uma vez que altas temperaturas de présinterização podem afetar a rugosidade do material usinado além de promover desgaste excessivo das ferramentas. Após a usinagem e aplicação de recobrimento no caso da coroa, o material é sinterizado acima de $1000^{\circ} \mathrm{C}$ a fim de conferir a resistência mecânica desejada [9].

Assim, o presente trabalho investigou a relação entre a microdureza e a temperatura de pré- 
sinterização de blocos de zircônia Y-TZP de fabricação experimental e seu comportamento ao ser submetido à usinagem. Os dados foram analisados por meio de ANOVA de um fator (one way) com $\mathrm{p} \leq 0,05$ e teste de Tukey-Kramer. A hipótese testada foi de que a diferença de $50^{\circ} \mathrm{C}$ na temperatura de pré-sinterização afeta a dureza embora o tempo de 2 e 4 horas não influencia significativamente a dureza do material. A maior temperatura de pré-sinterização testada possibilitou melhor comportamento na usinagem e dureza similar ao material comercial InCoris ZL - Sirona Dental Systems GmbH - Benshein, Alemanha.

\section{MATERIAIS E MÉTODOS}

Para o experimento foram utilizados 3 grupos experimentais e 2 grupos de procedência comercial, com base no material cerâmico zircônia Y-TZP que foram conformados em blocos pré-sinterizados e caracterizados pela microdureza, rugosidade superficial e em seguida usinados no formato de copings.

\subsection{Materiais}

Os 5 grupos são apresentados na Tabela 1, onde blocos representantes dos grupos foram denominados T1, T2, T3, ZL e ZC. Os blocos T1, T2 e T3 foram fabricados experimentalmente, nas dimensões 15,5 x 19,0 x 40,0 $\mathrm{mm}$, a partir do pó de zircônia, por meio de prensagem hidráulica uniaxial utilizando uma prensa Skay modelo $60 \mathrm{t}$ à pressão de $90 \mathrm{MPa}$ seguido de prensagem isostática (Prensa isostática de vasos gêmeos - LTC EESC - USP) [10] à temperatura ambiente $\left(27^{\circ} \mathrm{C}\right)$ à pressão de $200 \mathrm{MPa}$ e posteriormente seguiram para a pré-sinterização. Já os blocos ZL e ZC [11], de procedência comercial, nas mesmas dimensões, foram adquiridos comercialmente já pré-sinterizados e foram considerados como referenciais para a avaliação dos blocos experimentais durante o processo de usinagem.

Tabela 1: Descrição dos materiais utilizados nos ensaios.

\begin{tabular}{|c|c|c|c|}
\hline BLOCO & MATERIAL & FORNECEDOR & COMPOSIÇÃO \\
\hline T1 & \multirow{3}{*}{ Zircônia em pó } & \multirow{3}{*}{ MELox3Y-MELChemicals } & \multirow{3}{*}{$\begin{array}{c}\text { zircônia }\left(\mathrm{ZrO}_{2}\right) \text { estabilizada com } \\
5,0 \% \text { em peso de ítria }\left(\mathrm{Y}_{2} \mathrm{O}_{3}\right)\end{array}$} \\
\hline $\mathrm{T} 2$ & & & \\
\hline T3 & & & \\
\hline $\mathrm{ZL}$ & \multirow{2}{*}{$\begin{array}{l}\text { Zircônia em bloco } \\
\text { pré-sinterizado }\end{array}$} & $\begin{array}{l}\text { InCoris ZL - Sirona Dental Sys- } \\
\text { tems GmbH - Benshein, Alema- } \\
\text { nha }\end{array}$ & $\begin{array}{c}\mathrm{ZrO}_{2}+\mathrm{HfO}_{2}+\mathrm{Y}_{2} \mathrm{O}_{3} \geq 99,0 \% \\
\left(\mathrm{Y}_{2} \mathrm{O}_{3}>4,5-\leq 6,0 \%\right)\end{array}$ \\
\hline $\mathrm{ZC}$ & & Zircad - Ivoclar Vivadent Inc & $\begin{array}{c}95,00 \% \mathrm{ZrO}_{2}+5 \% \\
\left(\mathrm{HfO}_{2}+\mathrm{Al}_{2} \mathrm{O}_{3}+\mathrm{Y}_{2} \mathrm{O}_{3}\right)\end{array}$ \\
\hline
\end{tabular}

\subsection{Pré-sinterização}

Para a pré-sinterização foram determinadas as seguintes temperaturas: $\mathrm{T} 1$ a $900^{\circ} \mathrm{C}$ com patamar de 2 horas, $\mathrm{T} 2$ a $900{ }^{\circ} \mathrm{C}$ com patamar de 4 horas e $\mathrm{T} 3$ a $950^{\circ} \mathrm{C}$ com patamar de 2 horas.

\subsection{Preparação dos corpos de prova}

Os blocos (T1, T2, T3, ZL e ZC) foram fixados em um suporte metálico com godiva verde (composto de resina vegetal para uso odontológico) em bastão (Lysanda produtos odontológicos, São Paulo, SP, Brasil) e adaptados na cortadeira Isomet 1000 (Buehler, LakeBluff, IL, EUA), para a confecção de 1 corpo de prova (CP) para cada bloco, com as dimensões conforme determinado pela norma para ensaio de dureza Vickers em materiais cerâmicos (ASTM 1327-08) [12]. Em seguida os CPs foram embutidos em baquelite com a embutidora metalográfica EM30D (Teclago Indústria e Comércio Eireli-ME). A superfície das peças embutidas foram lixadas em uma sequência de lixas com granulometria 400, 500, 600, 800, 1200, 1500, 2000 e 2500 mesh e polidas em politriz metalográfica (Struers ApS) com pasta de diamante de granulometria $1 \mu \mathrm{m}$ e lubrificante a base de glicerina, com tempo de polimento de $20 \mathrm{~min}$. Para a limpeza da superfície polida foi utilizado álcool isopropílico $(99,8 \%)$.

Para que o acabamento da superfície proporcione a endentação acentuada ao submeter os CPs ao ensaio de microdureza Vickers, foi determinada a rugosidade Rq da superfície lixada e polida, utilizando o rugosímetro portátil Surtronic 3+ (Taylor Hobson Brasil), cujo comprimento da amostragem (cut-off) foi de $0,25 \mathrm{~mm}$, sendo obtidos 10 valores de rugosidade de cada copo de prova. 


\subsection{Ensaio de microdureza}

As medidas de microdureza Vickers foram realizadas nos próprios blocos, antes da confecção de prótese por usinagem. Foi utilizado o microdurômetro Mitutoyo HM-211, com as cargas de 0,1 a $1 \mathrm{kgf}$. Nas cargas de 0,1 e 0,5 kgf, as diagonais da impressão apresentaram assimetria, pontas deslocadas, cantos deformados e foram considerados não aceitáveis, conforme norma ASTM 1327-08 [12]. Assim foi definido a carga padrão de $1 \mathrm{kgf}$ para todos os CPs.

Devido à cor branca do material, foi necessário adicionar uma fonte externa de luz para a visualização da impressão na superfície produzida pelo penetrador de diamante (endentador). As diagonais do losango impressos na superfície foram medidas por meio de um microscópio acoplado à máquina de ensaio e a média foi calculada conforme a equação (1), sendo utilizadas para a determinação da microdureza Vickers $(H V)$, conforme a equação (2), que expressa a relação carga $(P)$, o ângulo entre as faces opostas da pirâmide de base quadrada $(\theta)$ e a média das diagonais $(d)[13]$.

$$
\begin{aligned}
& d=\left(d_{1}+d_{2}\right) / 2 \\
& H V=0,102\left[2 P \operatorname{sen}\left[(\theta / 2) / d^{2}\right]\right.
\end{aligned}
$$

O número de impressões obtidas foram 10 para cada material sendo o total de 50 . Para obter as imagens da impressão, os materiais submetidos ao ensaio de microdureza com as cargas de 0,1 a $0,5 \mathrm{kgf}$ foram analisadas pelo microscópio (Olympus modelo BX 56). Os materiais submetidos à carga de $1 \mathrm{kgf}$ foram submetidos ao recobrimento com plasma de ouro, em câmara de vácuo e analisado por microscopia eletrônica de varredura (MEV) com ampliação de 500X (Zeiss modelo EVO LS15).

\subsection{Teste de estabilidade na usinagem}

Para avaliar a resistência do bloco pré-sinterizado foi realizado o teste de estabilidade do material quando submetido aos esforços solicitados pelas ferramentas do centro de usinagem Cerec (Sirona, Charlotte, NC, USA) sendo os parâmetros relacionados na Tabela 2.

Tabela 2: Parâmetros de usinagem dos blanks.

\begin{tabular}{c|c}
\hline DENOMINAÇÃo & CARACTERÍSTICAS \\
\hline Tipo de fresa & Step Bur 12 S Cerec® (Ponta cilíndrica) \\
\hline $\begin{array}{c}\text { Tamanho de grão da ponta da } \\
\text { fresa }\end{array}$ & $64 \mu \mathrm{m}$ \\
\hline Velocidade de avanço da fresa & $1.0-1.5 \mathrm{~mm} / \mathrm{min}$ \\
\hline Frequência de rotação da fresa & $42.000 \mathrm{rpm}$ \\
\hline
\end{tabular}

Os blocos constituídos pelos materiais T1, T2 e T3 receberam acabamento com lixas em sequência de granulação 600, 800 e 1200 mesh.

Para o acoplamento do bloco no sistema de usinagem, um eixo flangeado de alumínio foi aderido com adesivo (Araldite ${ }^{\circledR}$ Hobby - Cola epóxi Brascola) e mantido em um molde durante 24 horas para a reação do adesivo.

Foi utilizado um sistema CAD/CAM composto por scanner para a digitalização do dente, software e centro de usinagem, para a confecção do coping. O processo iniciou-se com a digitalização do dente e a imagem obtida foi enviada ao programa computacional Sirona. A morfologia da prótese foi trabalhada pelo dentista o qual utilizou a imagem como base. Com a finalização do desenho do coping, os blocos de zircônia foram fixados no mandril do equipamento, foram fixadas as ferramentas do centro de usinagem Cerec e teve início o processo de usinagem. O fluido lubrificante utilizado foi água à temperatura ambiente, isento de adição de óleo ou qualquer produto químico. Para verificar a dimensão das paredes da prótese, foi utilizado um paquímetro universal.

\subsection{Exploração dos dados numéricos}

Os dados numéricos obtidos pelo ensaio de rugosidade e microdureza Vickers foram analisados por meio da estatística descritiva. A tendência central foi obtida pela média dos valores, porém, na presença de valores extremos, foram aplicados a mediana e os quartis. A variação ou quantidade de dispersão foi analisada por 
meio do cálculo da amplitude para estudar a dispersão total e amplitude interquartil para dispersão central. O cálculo do desvio padrão mostrou como os dados flutuam em torno da média, ou seja, a dispersão média em torno da média aritmética. A maneira de distribuição dos dados foi observada através do formato dos dados, onde foi mostrado o conjunto de dados simétricos e assimétricos. A forma gráfica dos dados foi demonstrada utilizando-se o gráfico de caixa, que representa a mediana, a amplitude interquartil, o valor mínimo e o máximo [14].

Para o teste de normalidade foi utilizado o método de Anderson-Darlin com $\mathrm{p}>0,05$ [15]. Para verificar a existência de diferenças estatísticas foi utilizado ANOVA de um fator [16], com o intervalo de confiança de $95 \%$ e teste de Tukey-Kramer, com nível de significância $0,05(\mathrm{p} \leq 0,05)[14]$.

\section{RESULTADOS}

3.1 Análises de superfície

A rugosidade média $(\mathrm{Rq})$ para cada grupo, desvio padrão e homogeneidade entre os grupos, está relacionada na Tabela 3, onde é possível perceber a tendência de similaridade entre as superfícies de T1 e T2 bem como entre as T3 e ZL. É observado que a rugosidade menor para o grupo T3 é devido à maior temperatura de présinterização onde se encontra um estágio mais avançado de formação de pescoços entre as partículas e início de rearranjo e formação dos grãos já na ocorrência de retração e densificação.

Tabela 3: Valores médios de rugosidade (Rq), tamanho da amostra (n), desvio padrão (DP) e grupos homogêneos (GP) de acordo com o teste de Tukey-Kramer.

\begin{tabular}{c|c|c|c|c}
\hline MATERIAL & $\mathbf{n}$ & RUGOSIDADE (Rq) & DP & GP \\
\hline T1 & 10 & 0,38 & 0,04 & $\mathrm{~A}$ \\
\hline $\mathrm{T} 2$ & 10 & 0,42 & 0,04 & $\mathrm{~A}$ \\
\hline $\mathrm{T} 3$ & 10 & 0,28 & 0,04 & $\mathrm{~B}$ \\
\hline ZL & 10 & 0,24 & 0,04 & $\mathrm{~B}$ \\
\hline ZC & 10 & 0,15 & 0,03 & $\mathrm{C}$ \\
\hline
\end{tabular}

O gráfico de intervalos (Figura 1) mostra que, embora todas as médias apresentem valores distintos, provavelmente, entre T1 e T2, as diferenças são provavelmente não significativas uma vez que a barra dos intervalos de confiança se sobrepõe. O mesmo ocorre com T3 e ZL, indicando a mesma a qualidade superficial.

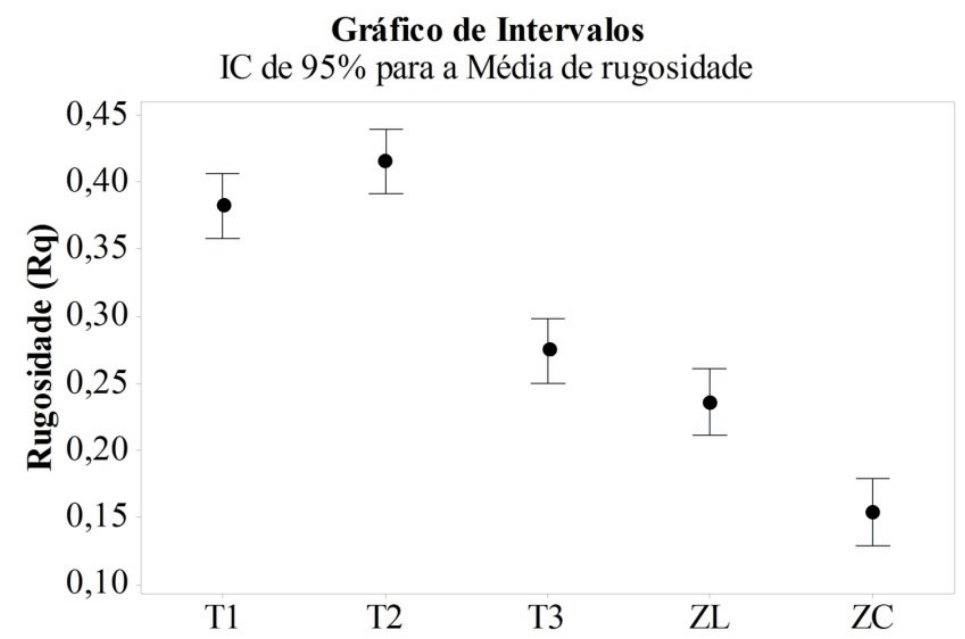

Figura 1: Gráfico de intervalos com os valores médios de rugosidade.

As hipóteses apresentadas no gráfico de intervalos são reforçadas nas comparações múltiplas de Tukey-Kramer, Figura 2, onde T1 e T2 não apresentaram diferenças estatísticas significativas na qualidade superficial e o mesmo ocorreu com ZL e T3. 
Teste de Tukey-Kramer

Diferença ente as Médias de rugosidade

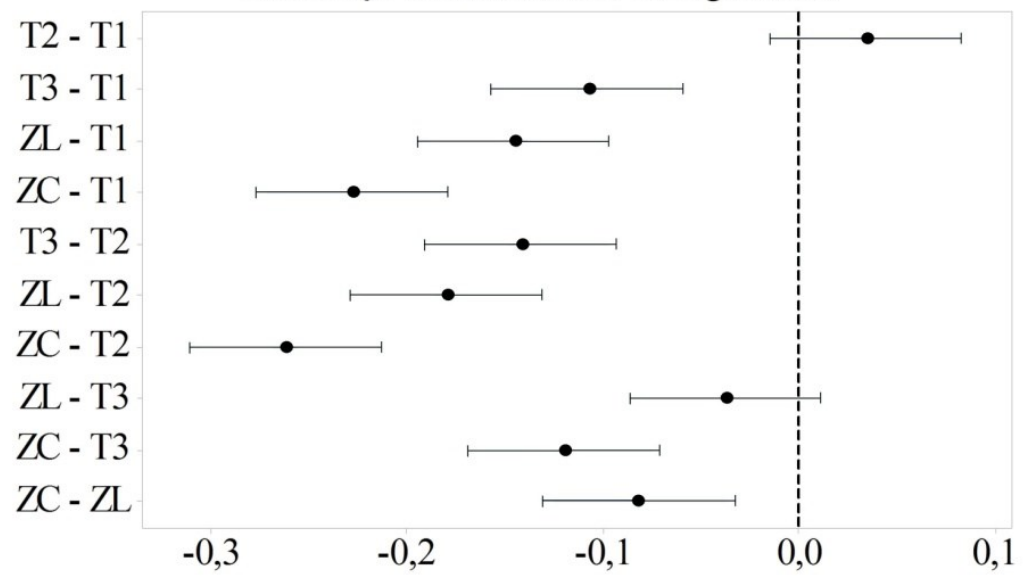

Figura 2: Múltiplas comparações de Tukey-Kramer para a rugosidade.

\subsection{Microdureza}

Ao aplicar a carga de 1 kgf, a impressão resultante na superfície de todos os CPs (T1, T2, T3, ZL e ZC) foi o losango conforme descrito na norma ASTM 1327-08 [12]. A Figura 3 mostra a impressão, na superfície de $\mathrm{T} 2$, isenta de faces e contornos irregulares que possam comprometer a precisão e exatidão da medida.

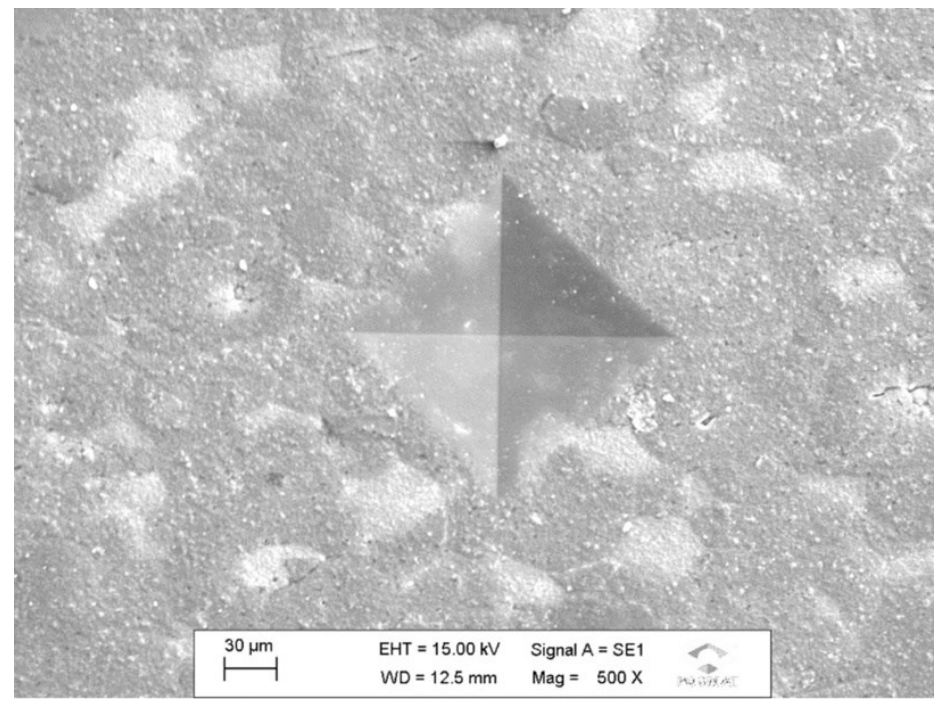

Figura 3: Impressão típica na superfície do bloco T2 causada pelo penetrador piramidal do ensaio de microdureza Vickers.

$\mathrm{Na}$ Tabela 4 está relacionada a média de microdureza Vickers (HV 1), desvio padrão DP e os grupos homogêneos de acordo com o teste de Tukey-Kramer, dos CPs T1, T2, T3, ZL e ZC. A Tabela 5 mostra a diferença de média entre os grupos e o nível de significância ou valor $\mathrm{p} \leq 0,05$ para a hipótese nula de igualdade.

Tabela 4: Valores médios de microdureza Vickers HV 1, desvio padrão (DP), tamanho da amostra (n) e grupos homogêneos de acordo com o teste de Tukey-Kramer.

\begin{tabular}{c|c|c|c|c}
\hline MATERIAL & $\mathbf{n}$ & HV 1 & DP & GP \\
\hline T1 & 10 & 49,67 & 1,8 & C \\
\hline T2 & 10 & 51,00 & 2,4 & C \\
\hline T3 & 10 & 55,50 & 1,0 & B \\
\hline ZL & 10 & 56,20 & 1,8 & B \\
\hline ZC & 10 & 63,20 & 1,1 & A \\
\hline
\end{tabular}


Tabela 5: Diferença de média entre os grupos e valor $\mathrm{p}$.

\begin{tabular}{c|c|c}
\hline GRUPOS & DIFERENÇA DE MÉDIAS & VALOR $\mathbf{p}$ \\
\hline $\mathrm{T} 2-\mathrm{T} 1$ & 1,29 & 0,493 \\
\hline $\mathrm{T} 3-\mathrm{T} 1$ & 5,78 & 0,000 \\
\hline ZL - T1 & 6,48 & 0,000 \\
\hline ZC - T1 & 13,54 & 0,000 \\
\hline T3 - T2 & 4,49 & 0,000 \\
\hline ZL - T2 & 5,19 & 0,000 \\
\hline ZC - T2 & 12,25 & 0,000 \\
\hline ZL - T3 & 0,7 & 0,903 \\
\hline ZC - T3 & 7,76 & 0,000 \\
\hline
\end{tabular}

Aplicando-se ANOVA para todos os grupos, considerando-se o intervalo de confiança de 95\%, verificou-se a existência de diferenças ente os grupos pesquisados.

No gráfico de caixa da Figura 4 e na Tabela 4, é possível observar a distribuição dos valores de microdureza, no qual o material experimental que apresentou o valor mais elevado foi T3 e o maior valor entre os comerciais foi o ZC. Também pode ser constatado que existe a sobreposição na barra da amplitude, entre T1 e T2 ocorrendo o mesmo com T3 e ZL. O teste de Tukey-Kramer (Figura 5) confirmou essa hipótese onde esses grupos se encontram na região zero, ou seja, hipótese nula de diferenças entre os grupos. A região onde os demais materiais não se encontram na região zero sugere a existência de diferenças entre os grupos.

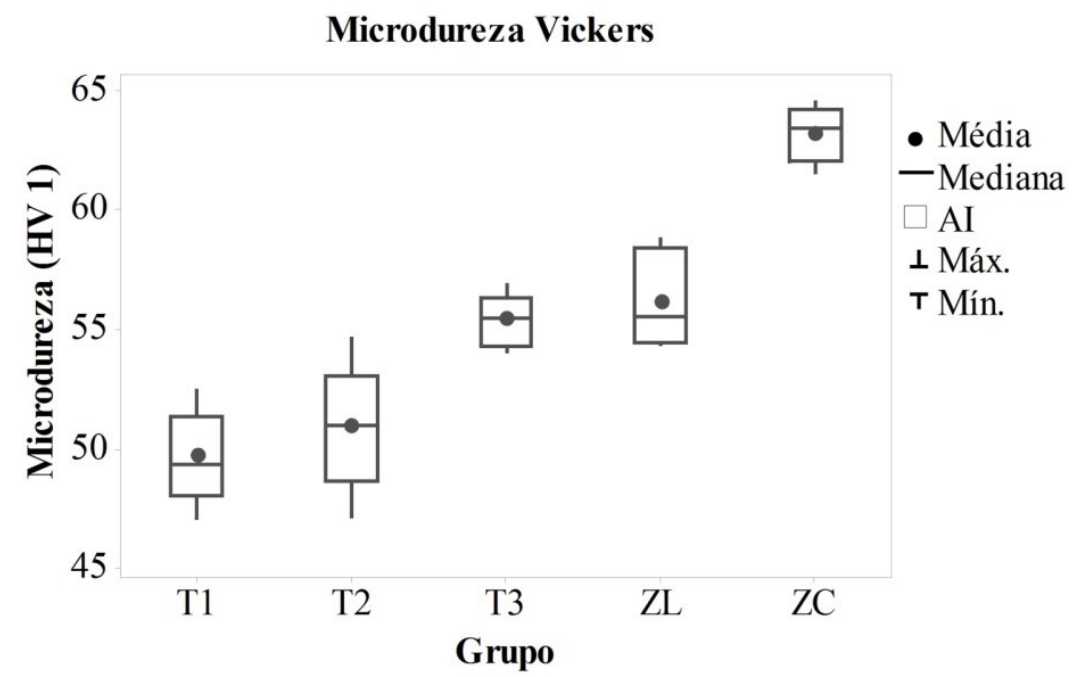

Figura 4: Distribuição dos valores médios de microdureza Vickers (HV1), amplitude interquartil (AI) e valores mínimos e máximos (amplitude) dos materiais T1, T2, T3, ZC e ZL (n-10). 


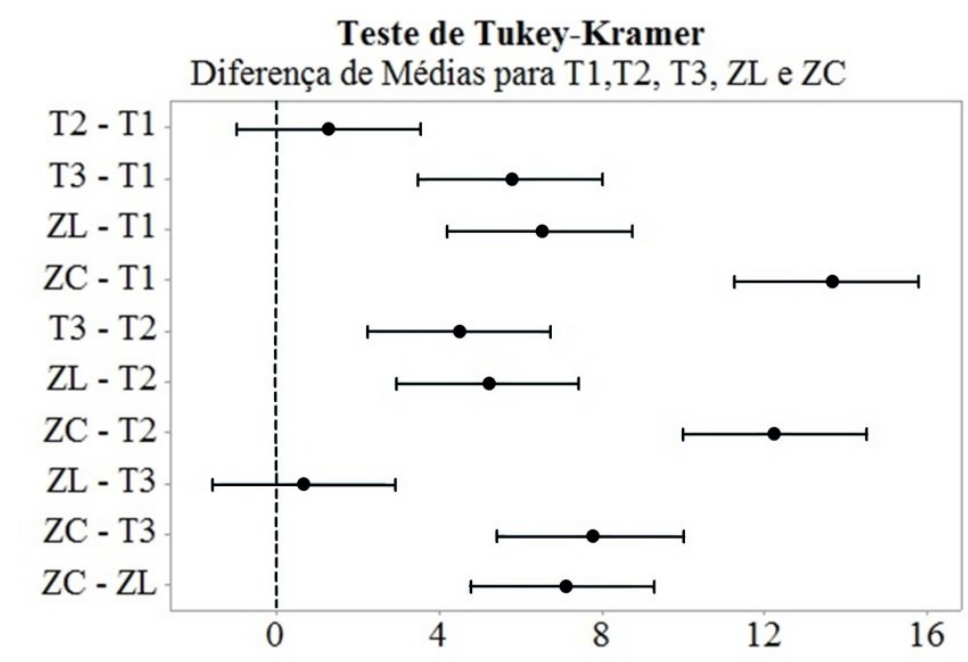

Figura 5: Testes Simultâneos de Tukey-Kramer para as diferenças de médias entre T1, T2, T3, ZC e ZL (n-10).

\subsection{Confecção dos implantes}

Os copings apresentaram uma estrutura em forma de casca, ou seja, apesar de ser um elemento estrutural, suas paredes são finas (Figura7), aproximadamente $0,6 \mathrm{~mm}$ na forma pré-sinterizada. Essas paredes resistiram aos esforços provocados pelas ferramentas de usinagem, resultando em próteses com estabilidade dimensional e morfológica. Os blocos T1, T2 e T3 não apresentaram problemas durante a usinagem, resultando em coping livres de trincas ou deformações. A Figura 6 mostra o material T3 submetido à ação da ferramenta de fresamento, apresentando estabilidade ao passar pelo processo de usinagem, com intensa lubrificação.
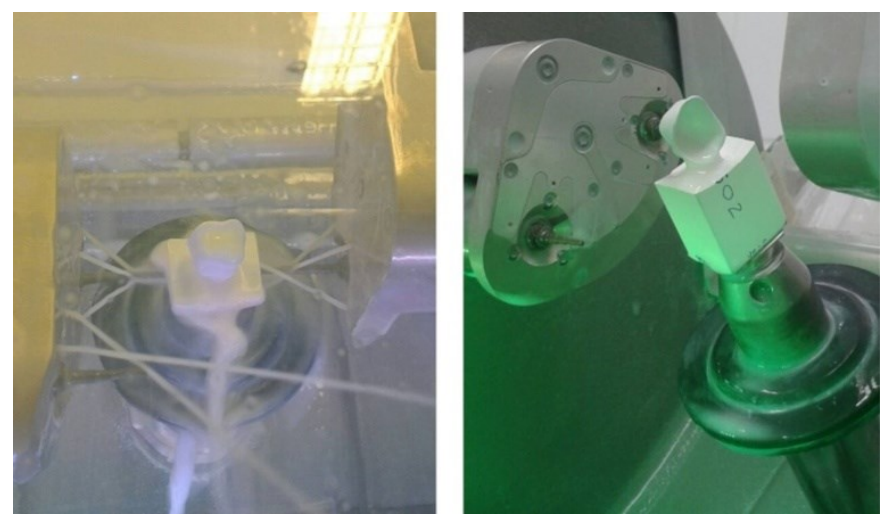

Figura 6: Confecção de coping para implante odontológico, com o bloco T3, por CAD/CAM.
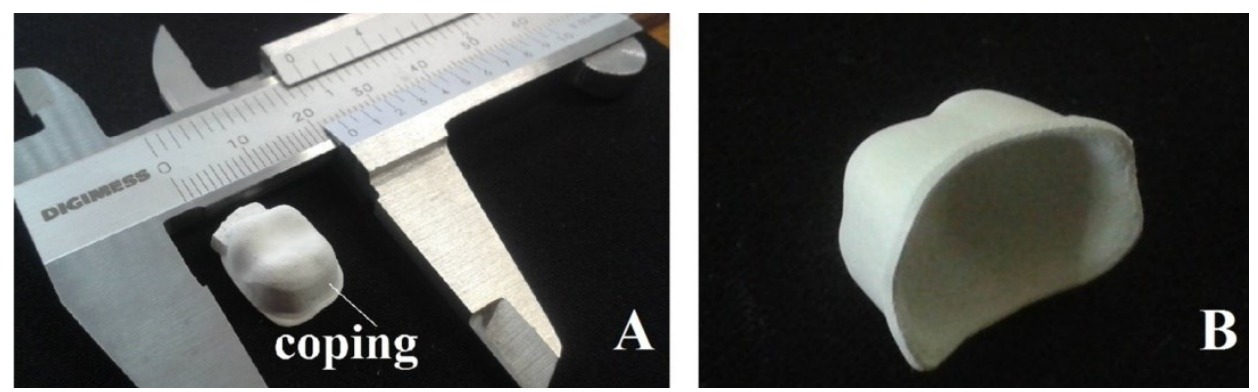

Figura 7: Coping confeccionado com o material T3: (A) morfologia do dente e (B) estrutura interna em forma de casca. 


\section{DISCUSSÃO}

Com respeito à medida de microdureza Vickers em cerâmicas avançadas, um dos requisitos para a obtenção de impressão em formato losango regular é o acabamento da superfície do corpo de prova, que deve ser lixada e polida para garantir a rugosidade necessária, a fim de evitar uma impressão deformada e rejeição da medida.Durante a revisão bicliográfica, não foi possível encontrar uma metodologia padronizada para a medida de microdureza da zircônia pré-sinterizada na temperatura utilizada neste trabalho. Assim o procedimento foi baseado na norma para ensaio de microdureza Vickers para cerâmicas avançadas totalmente sinterizadas [11], que sugere a rugosidade de $0,1 \mu \mathrm{m}$ Rq quando o revestimento ou tratamento da superfície do material investigado permitir essa condição. Os valores médios de rugosidade obtidos na presente pesquisa (entre $0,15 \mathrm{e}$ $0,42 \mu \mathrm{m} \mathrm{Rq}$ ) apresentaram-se superiores uma vez que o material se encontra em estágio anterior ao do processo de transformação total. As temperaturas utilizadas nos blocos experimentais podem estar de acordo com a hipótese relatada por LI et al. [17], onde, em torno de $900^{\circ} \mathrm{C}$, a zircônia se encontra em processo intermediário ao da sinterização total, com o rearranjo dos grãos e baixa taxa de retração, estando sujeito à presença de poros. Neste trabalho, os blocos experimentais $\mathrm{T} 1, \mathrm{~T} 2$ e T3, pré-sinterizados abaixo de $1000^{\circ} \mathrm{C}$, não alcançaram a máxima densificação, uma vez que a essa característica é encontrada em materiais sinterizados acima de $1180^{\circ} \mathrm{C}$ [18]. Assim a rugosidade pode estar relacionada à presença desses poros. Contudo os blocos apresentaram acabamento superficial uniforme onde foi possível realizar a impressão de microdureza Vickers com losango de diagonais isentas de deformação. Dessa forma, assume-se que a rugosidade alcançada com o lixamento e polimento não exerceram influencia significativa na endentação.

As análises estatísticas dos resultados de rugosidade indicaram que as endentações na superfície de T1 e T2 foram realizadas em materiais com a mesma qualidade superficial, o mesmo ocorreu entre T3 e ZL.

Os resultados de microdureza Vickers indicaram que o tempo de pré-sinterização ( 2 e 4 horas) à temperatura de $900^{\circ} \mathrm{C}$ não influenciou a microdureza do material, porém foi significativo com o aumento de $50^{\circ} \mathrm{C}$. Uma das limitações das análises comparativas foi a ausência de referências de microdureza de zircônia Y-YZP pré-sinterizados a diferentes temperaturas. Mas nas investigações de DURAN[18], relacionado ao comportamento da microestrutura da zircônia sinterizada à baixas temperaturas, é relatado que ocorre uma baixa taxa de densificação até $800^{\circ} \mathrm{C}$, denominada como primeiro estágio, e no segundo estágio, entre $800 \mathrm{e}$ $1180^{\circ} \mathrm{C}$ a densidade relativa aumenta linearmente, porém com baixa taxa de energia de ativação, caracterizando a difusão no contorno de grão como o principal mecanismo de densificação. Esse comportamento microestrutual pode explicar a diferença de microdueza ente T3 e T2 e a hipótese de igualdade entre T1 e T2.

O maior nível de dureza implica em maior desgaste da ferramenta de usinagem, sendo significativo quando materiais mais duros são usinados o que não ocorre com os materiais mais macios [19].

Pesquisas $[20,21,22]$ indicam que o material comercial ZL estatisticamente similar a T3, normalmente utilizado para usinar componentes de próteses odontológicas em sistemas $\mathrm{CAD} / \mathrm{CAM}$, proporciona co- $^{-}$ pings de morfologia e resistência mecânica adequada à aplicação.

Embora, as forças geradas entre a ferramenta de diamante e os blocos de zircônia Y-TZP présinterizados (T1, T2 e T3) durante o fresamento [23] no centro de usinagem não tenham afetado a estabilidade do material bem como os copings resultantes do processo, o bloco T3 apresentou resistência mecânica mais próxima ao material comercial ZL. Assim, as variáveis do processo de fabricação experimental de T3, bem como a composição química proporcionaram um bloco com as características necessárias para a usinagem de copings para próteses odontológicas.

\section{CONCLUSÕES}

Os resultados dos ensaios de microdureza obtidos no presente trabalho podem contribuir para estimar o desempenho da usinagem bem como a estabilidade dos blocos durante o processo de usinagem dos copings.

Diante das análises estatísticas realizadas por ANOVA, com índice de confiabilidade de 95\%, utilizando os dados dos ensaios de microdureza Vickers, foi demonstrado que a diferença de $50^{\circ} \mathrm{C}$ na temperatura de pré-sinterização, acima de $900^{\circ} \mathrm{C}$, afetou a microdureza, contudo o tempo de 2 e 4 horas não exerceu influência significativa. Assim, a temperatura de pré-sinterização representa um parâmetro de processo significativo que pode influenciar a resistência mecânica dos blocos pré-sinterizados bem como a qualidade das peças usinadas.

O presente trabalho apresentou um processo de fabricação experimental de blocos de zircônia Y-TZP, com três processos térmicos de pré-sinterização distintos, para a usinagem de copings para prótese em sistema $\mathrm{CAD} / \mathrm{CAM}$ odontológico. $\mathrm{O}$ processo térmico com patamar à $950^{\circ} \mathrm{C}$ por 2 horas foi mais adequado à 
fabricação de copings uma vez que proporcionou ao bloco características mecânicas (rugosidade e microdureza) similares ao bloco comercial ZL normalmente utilizado na usinagem de coping para a odontologia. A microdureza média de 55,50 HV 1 deste conjunto de blocos experimentais, proporcionou resistência mecânica aos esforços solicitados pelas ferramentas de corte e lubrificação, sem prejudicar o processo de usinagem, resultando em peças isentas de deformação.

\section{AGRADECIMENTOS}

Os autores agradecem à CAPES pelo apoio financeiro para o desenvolvimento deste trabalho e aos profissionais do Laboratório de Materiais da Faculdade de Engenharia de Bauru da Universidade Estadual Paulista Julio de Mesquita Filho, Laboratório de Tribologia e Compósitos da Escola de Engenharia de São Carlos da Universidade de São Paulo, Laboratório de Materiais e Denstística da Faculdade de Odontologia de Bauru da Universidade de São Paulo e Laboratório de Relaxações Anelásticas da Faculdade de Ciências de Bauru da Universidade Estadual Paulista.

\section{BIBLIOGRAFIA}

[1] TAMAKI, Y., HOTTA, Y., KUNII, J., et al., "CAD/CAM All Ceramic Dental Restorations on Implants: A Panacea or a Challenge?”, Dental medicine research, v. 30, n. 1, pp. 42-49, Mar. 2010.

[2] MARCHACK, B. W., FUTATSUKI, Y., MARCHACK, C. B., et al., "Customization of milled zirconia copings for all-ceramic crowns: a clinical report", The Journal of Prosthetic Dentistry, v. 99, n. 3, pp. 169176, Mar. 2008.

[3] ARNETZL, G. V., ARNETZL, G., "Die CAD/CAM-Abutmentkrone Biokompatible und ästhetische Restauration auf Implantaten", Stomatologie, v. 110, n. 3, pp. 19-24, Maio 2013.

[4] PROTOPAPADAKI, M., MONACO, E. A., KIM, H., et al., "Comparison of fracture resistance of pressable metal ceramic custom implant abutment with a commercially fabricated CAD/CAM zirconia implant abutment", The Journal of Prosthetic Dentistry, v. 110, pp. 389-396, Nov. 2013.

[5] SANTOS, M. J., COSTA, M. D., RUBO, J. H., et al., "Current all-ceramic systems in dentistry: a review", Compendium of Continuing Education in Dentistry, v. 36, n. 1, pp. 31 -7, Jan. 2015.

[6] SALVENDY, G. Handbook of Industrial Engineering: Technology and Operations Management, 3 ed., Hoboken, NJ, USA, John Wiley \& Sons, 2007.

[7] WESTERHEIDE, R., DRÜSEDAU, K. A., HOLLSTEIN, T., et al., "Advances in Characterisation of Machined Green Compacts", Journal of the European Ceramic Society, v. 17, n. 2, pp. 467-472, Ago. 1997.

[8] MOHANTY, S., RAMESHBABU, A. P., MANDAL, S., et al., "Critical issues in near net shape forming via green machining of ceramics: A case study of alumina dental crown", Journal of Asian Ceramic Societies, v. 1, n. 3, pp. 274-281, Jul. 2013.

[9] DENRY, I., KELLY, J. R., "State of the art of zirconia for dental applications", Dental Materials, v. 24, n. 3, pp. 299-307, Mar. 2008.

[10] FORTUlAN, C. A., PENAZI, L. A., PEDROSO, M. P. G., "Twin vessel isostatic press: design", Cerâmica, v. 60, n. 354, pp. 199-204, Jun. 2014.

[11] RAMOS, C. M., CESAR, P. F., BONFANTE, E. A., et al., "Fractographic principles applied to Y-TZP mechanical behavior analysis", Journal of the mechanical behavior of biomedical materials, v. 55, pp. 215223, 2016.

[12] ASTM, C., "1327-08. Standard test method for Vickers indentation hardness of advanced ceramics", Annual Book of ASTM Standards, 2008.

[13] GARCIA, A., SPIM, J. A., SANTOS, C. A., Ensaios dos Materiais, 2. ed., Rio de Janeiro, LTC, 2014.

[14] LEVINE, D. M., BERENSON, M. L., STEPHAN, D., Estatística: teoria e aplicações-usando Microsoft Excel português, Rio de Janeiro, LTC, 2005.

[15] MORAES, C. F., FERREIRA, J. R., BALESTRASSI, P. P., "Análise crítica da aplicação de métodos estatísticos em processos definidos por dados que não apresentam distribuição normal", Gepros: Gestão da Produção, Operações e Sistemas, v. 1, n. 1, pp. 7, Abr. 2006.

[16] ALAO, A.R., YIN, L., "Loading rate effect on the mechanical behavior of zirconia in nanoindentation", Materials Science and Engineering: A, v. 619, pp. 247-255, Dez. 2014.

[17] LI, J. Z., WU, T., YU, Z. Y., et al., "Micro machining of pre-sintered ceramic green body", Journal of Materials Processing Technology, v. 212, n. 3, pp. 571-579, Mar. 2012. 
[18] DURAN, P., VILLEGAS, M., CAPEL, F., et al., "Low-temperature sintering and microstructural development of nanocrystalline Y-TZP powders", Journal of the European Ceramic Society, v. 16, n. 9, pp. 945952, Jan. 1996.

[19] LEBON, N., TAPIE, L., VENNAT, E., et al., "Influence of CAD/CAM tool and material on tool wear and roughness of dental prostheses after milling", The Journal of prosthetic dentistry, v. 114, pp. 236-247, Ago. 2015.

[20] GOMES, E. A., ASSUNÇÃO, W. G., ROCHA, E. P., et al., "Cerâmicas odontológicas: o estado atual”, Cerâmica, v. 54, pp. 319-325, 2008.

[21] GUESS, P. C., ATT, W., STRUB, J. R., “Zirconia in Fixed Implant Prosthodontics", Clinical Implant Dentistry and Related Research, v. 14, pp. 633-645, 2012.

[22] SANTOS, M. J. M. C., COSTA, M. D., RUBO, J. H., et al., "Current All-Ceramic Systems in Dentistry: A Review”, Compedium continuing education 2. Ceramic System, v. 36, n. 1, 2015

[23] LUTHARDT, R. G. R. G., HOLZHÜTER, M. S., RUDOLPH, H., et al., "CAD/CAM-machining effects on Y-TZP zirconia”, Dental Materials, v. 20, pp. 655-662, Set. 2004. 